\title{
Multi-modal treatment strategy for achieving an aesthetic lower face
}

\author{
Tae Kwang Jeong ${ }^{1}$, Chang Ho Chung ${ }^{2}$, Kyung Hee Min ${ }^{2}$ \\ ${ }^{1}$ HB Plastic Surgery Clinic, Seoul; ${ }^{2}$ Department of Plastic and Reconstructive Surgery, Nowon Eulji Medical Center, Eulji University School of \\ Medicine, Seoul, Korea
}

Background Most women consider an oval-shaped face to be youthful and beautiful. In recent years, demand has grown for surgical procedures with a shorter downtime and fewer complications. These minimally invasive procedures include botulinum toxin type A (BoNTA) injection, filler injection, suction-assisted liposuction (SAL), laser-assisted lipolysis (LAL), thread lifting, and fat grafting. This study aims to introduce an effective method for creating an aesthetically pleasing lower face using a combination of minimally invasive procedures.

Methods From March 2017 to March 2019, 94 patients simultaneously underwent LAL, SAL, and thread lifting. Ancillary procedures such as BoNTA injections, hyaluronic acid filler injections, and removal of the buccal fat pad (BFP) were selectively performed according to the patient's condition.

Results Patients rated their postoperative satisfaction as very satisfied, satisfied, dissatisfied, or very dissatisfied. Approximately $83 \%$ of all respondents were satisfied with the results, whereas the remaining respondents had complaints regarding the outcomes. The most common reasons for dissatisfaction were a longer-than-expected recovery time and undercorrection, and the most severe complaint was skin depression as a result of overcorrection.

Conclusions Our method of simultaneously performing LAL, SAL, and thread lifting, while adding BoNTA, filler injections, and BFP removal as needed, was capable of producing consistent and reliable aesthetic outcomes for the lower face.

Keywords Esthetics / Face / Minimally invasive surgical procedures

\author{
Correspondence: Kyung Hee Min \\ Department of Plastic and \\ Reconstructive Surgery, Nowon Eulji \\ Medical Center, Eulji University School \\ of Medicine, 68 Hangeulbiseong-ro, \\ Nowon-gu, Seoul 01830, Korea \\ Tel: +82-2-970-8255 \\ Fax: +82-2-978-4772 \\ E-mail:mkh797@hanmail.net
}

This article was presented at PRS Korea on November 8-10, 2019, in Seoul, Korea.

\section{INTRODUCTION}

Most women prefer a slim and softly curved jawline, and consider an oval-shaped face, as seen from the front, to be youthful and beautiful. In contrast, faces with a wide lower area or a strongly-curved jawline are considered unappealing. The conventional workhorse for aesthetic enhancement of the lower face is the facelift; however, demand is growing for surgical pro- cedures with a shorter downtime that avoid the complications of invasive techniques, such as facelift procedures [1]. Common minimally invasive methods to make the lower face youthful and attractive include botulinum toxin type A (BoNTA) injection, filler injection, suction-assisted liposuction (SAL), laserassisted lipolysis (LAL), thread lifting, and fat grafting. A cosmetic surgeon needs to explore methods that satisfy patients' expectations, while minimizing complications and downtime. 
To do so, it is necessary to understand and become familiar with minimally invasive procedures and appropriately combine them to produce optimal results for each patient. The aim of this study was to introduce an effective method for creating an aesthetically pleasing lower face using a combination of minimally invasive procedures.

\section{METHODS}

Between March 2017 and March 2019, 94 patients simultaneously underwent LAL, SAL, and thread lifting. BoNTA injections, hyaluronic acid filler injections, and removal of the buccal fat pad (BFP) were selectively performed according to patients' condition (Table 1).

The patients were between 17 and 67 years of age (mean age, 39.2 years), including 87 women and seven men. Patients with a history of facial surgery using a large incision, such as surgical procedures involving the bone, chin implant insertion, and facelift, were excluded. Informed consent was obtained from each patient, and the study was performed in accordance with the ethical standards of the Declaration of Helsinki.

\section{Preoperative markings}

All designs were made with the patient in the sitting position. With a blue marker, the lower border of the mandible, prejowl

\section{Table 1. Patient's distribution by procedures}

\section{Used procedures}

$L A L+S A L+$ thread lift

$L A L+S A L+$ thread lift + BoNTA injection

$L A L+S A L+$ thread lift + HA filler injection

$L A L+S A L+$ thread lift + BoNTA \& HA filler injection

$L A L+S A L+$ thread lift + BFP removal

$L A L+S A L+$ thread lift + BFP removal + BoNTA injection

LAL, laser-assisted lipolysis; SAL, suction-assisted liposuction; BoNTA, botulinum toxin type $\mathrm{A} ; \mathrm{HA}$, hyaluronic acid; BFP, buccal fat pad. sulcus, median line of the face, and cervicomental crease were marked, and a line was drawn to connect the caudal margins of the earlobe and the ipsilateral oral commissure. With a red marker, the lipodystrophic areas of the lower face were marked using a contour line pattern. Patients were asked to make facial expressions to observe volumetric changes in the soft tissue, and the design was changed if there were areas from which fat should not be removed or should be conservatively removed. The direction of thread lifting was determined by pulling the facial skin toward the posterosuperior direction. After marking the location at the distal end, the insertion site of the thread was marked through the temporal hairline using a black marker. The design was made such that the distal end of the thread was at least $1.5 \mathrm{~cm}$ away from the prejowl sulcus and the lower border of the mandible (Fig. 1).

\section{Operative methods}

All procedures were performed under intravenous sedation.

\section{LAS and SAL}

Three stab incisions were made at the caudal margins of both earlobes and the midpoint of the submental crease, followed by administration of a tumescent solution with a $1.5-\mathrm{mm}$ cannula. The tumescent solution was prepared by mixing $200 \mathrm{~mL}$ of normal saline, $20 \mathrm{~mL}$ of $2 \%$ lidocaine, and $0.5 \mathrm{~mL}$ of 1:1,000 epinephrine. Then, $30 \mathrm{~mL}$ was administered to the ipsilateral lower face and submandibular area, and $20 \mathrm{~mL}$ to the submental area (mean, $80 \mathrm{~mL}$; range, $60-110 \mathrm{~mL}$ ). After 15 minutes, a 1,444$\mathrm{nm}$ neodymium-doped yttrium aluminum garnet (Nd:YAG) laser cannula (Accusculpt; Lutronic Corp., Goyang, Korea) was inserted into the subcutaneous layer through the incision site, and lipolysis was performed. The procedure was performed by retracting the cannula tip at $1 \mathrm{~cm} / \mathrm{sec}$ at approximately $400 \mathrm{~J}$ in the ipsilateral lower face and submandibular area and approximately $200 \mathrm{~J}$ in the submental area (mean, 1,000 J; range, 700$1,500 \mathrm{~J}$ ). The parameters were set to a pulse rate of $25 \mathrm{~Hz}$, a

Fig. 1. Preoperative markings

Frontal (A), worm's eye (B), and oblique views (C).
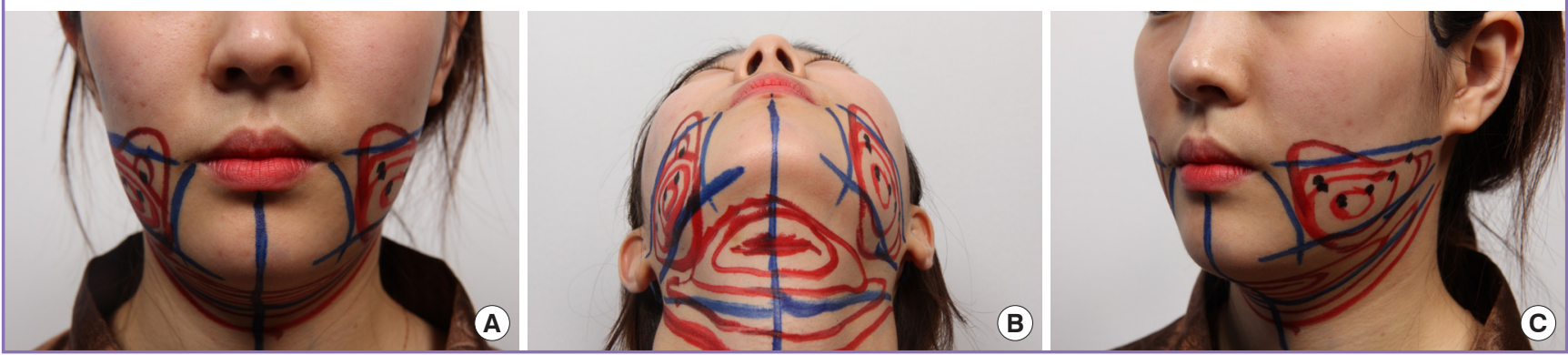
pulse energy of $200 \mathrm{~mJ}$, a pulse width of $100 \mu \mathrm{s}$, and power of $4 \mathrm{~W}$. After completing LAL, SAL was performed along the preoperative design using a 2.0-mm cannula.

\section{Thread lifting}

After liposuction, five insertion sites were made by punctures along the temporal hairline using an 18-gauge needle for thread lifting. Through each puncture site, a cannula containing a 20cm 1-0 barbed polydioxanone (PDO) thread (Ultra V Ez Cannula; Ultra V Co., Ltd., Seoul, Korea) was inserted. The cog portion of this thread is $10 \mathrm{~cm}$, and the cogs form a $360^{\circ}$ spiral. While placing the thread in the deep plane of the subcutaneous layer, it was ensured that the distal end of the thread did not create any dimple or linear depression on the skin. After the distal end of the thread reached the preoperative target point, the facial soft tissue was retracted posterosuperiorly and the cannula was smoothly removed. While removing the cannula, the path of the thread was gently compressed with the palm of the other hand to promote close contact between the tissue and thread barb. After inserting five threads on one side, they were cut while pulling the proximal stump of the thread, to prevent the proximal end from protruding. If the skin was dimpled, the site was lightly massaged immediately after the procedure.

\section{BoNTA and hyaluronic acid filler injections}

If masseter hypertrophy was observed upon physical examina- tion, 20 IU of BoNTA (Neuronox; Medytox Inc., Ochang, Korea) was injected into each masseter immediately before surgery. For patients with inadequate chin projection, or a prominent prejowl sulcus, an hyaluronic acid filler (Restylane Lyft; Galderma, Uppsala, Sweden) was injected after finishing the thread lifting procedure.

\section{BFP removal}

In patients with full jowls and a plump buccal region, the BFP was removed if superficial fat was not felt on palpation and if the middle cheek compartment was not hollow. BFP removal was performed conservatively through a $1-\mathrm{cm}$ intraoral incision.

\section{RESULTS}

Follow-up examinations were performed at 1 month and 6 months postoperatively (Figs. 2, 3).

Prolonged edema, skin dimpling or depression, thread protrusion, and overcorrected or undercorrected lipodystrophy were considered as postoperative complications. Seroma or hemato$\mathrm{ma}$ and visible scars were not observed in any patients (Table 2 ).

A satisfaction survey was conducted either face-to-face $(\mathrm{n}=$ 17) or over the phone $(n=37)$ at a minimum of 6 months after surgery. Patients rated their satisfaction levels as very satisfied, satisfied, dissatisfied, or very dissatisfied (Table 3). The most common reasons for dissatisfaction were a longer-than-expected

\section{Fig. 2. A case of multi-modal treatment}

A 25-year-old female patient underwent laser-assisted lipolysis, suction-assisted liposuction, thread lifting, buccal fat pad removal, and botulinum toxin type $A$ injection into the masseter muscle. Preoperative (A-C) and postoperative 6 months (D-F).

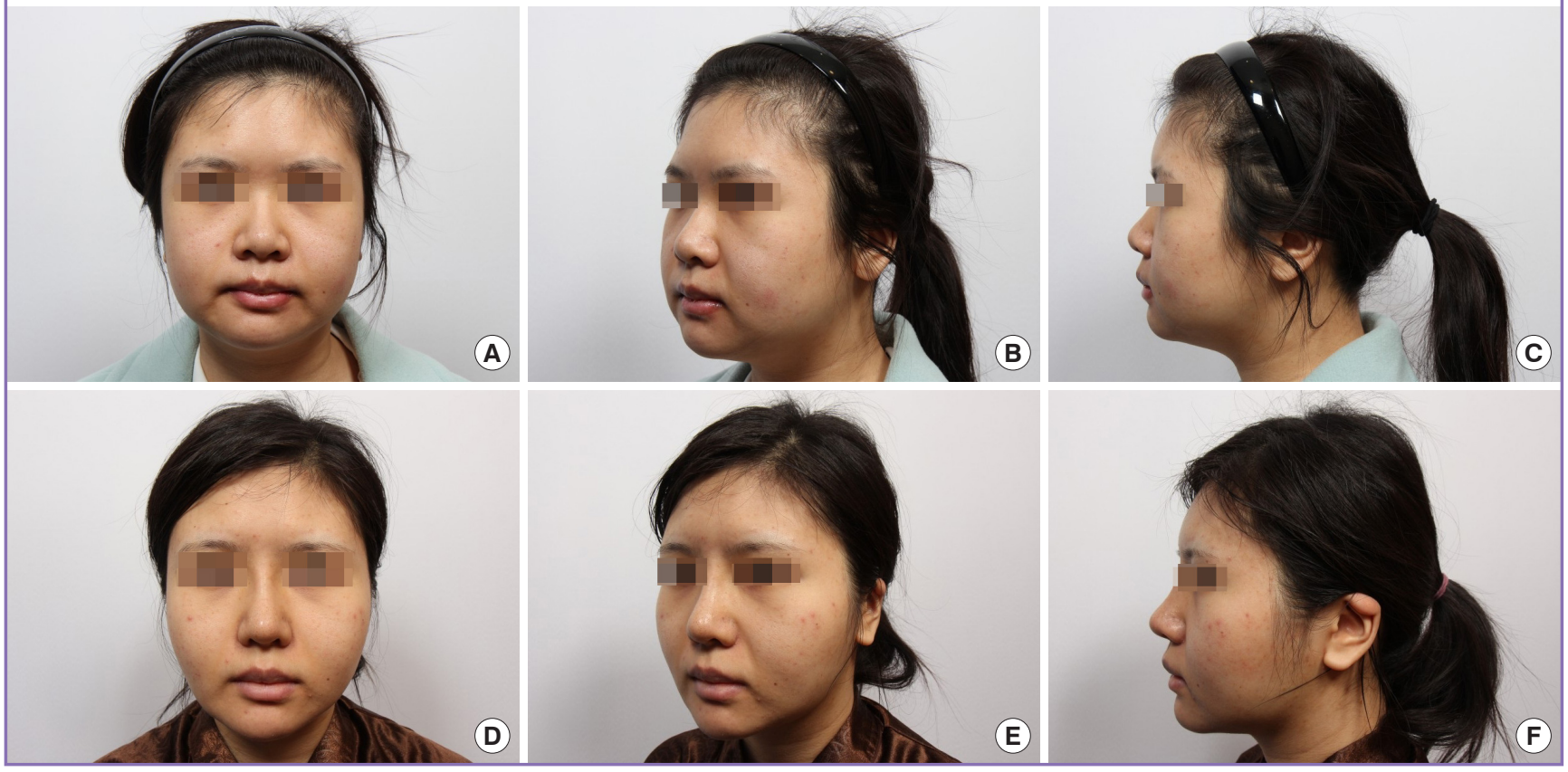




\section{Fig. 3. A case of multi-modal treatment}

A 42-year-old female patient underwent laser-assisted lipolysis, suction-assisted liposuction, thread lifting, and botulinum toxin type A injection into the masseter muscle. Preoperative (A-C) and postoperative 8 months (D-F).
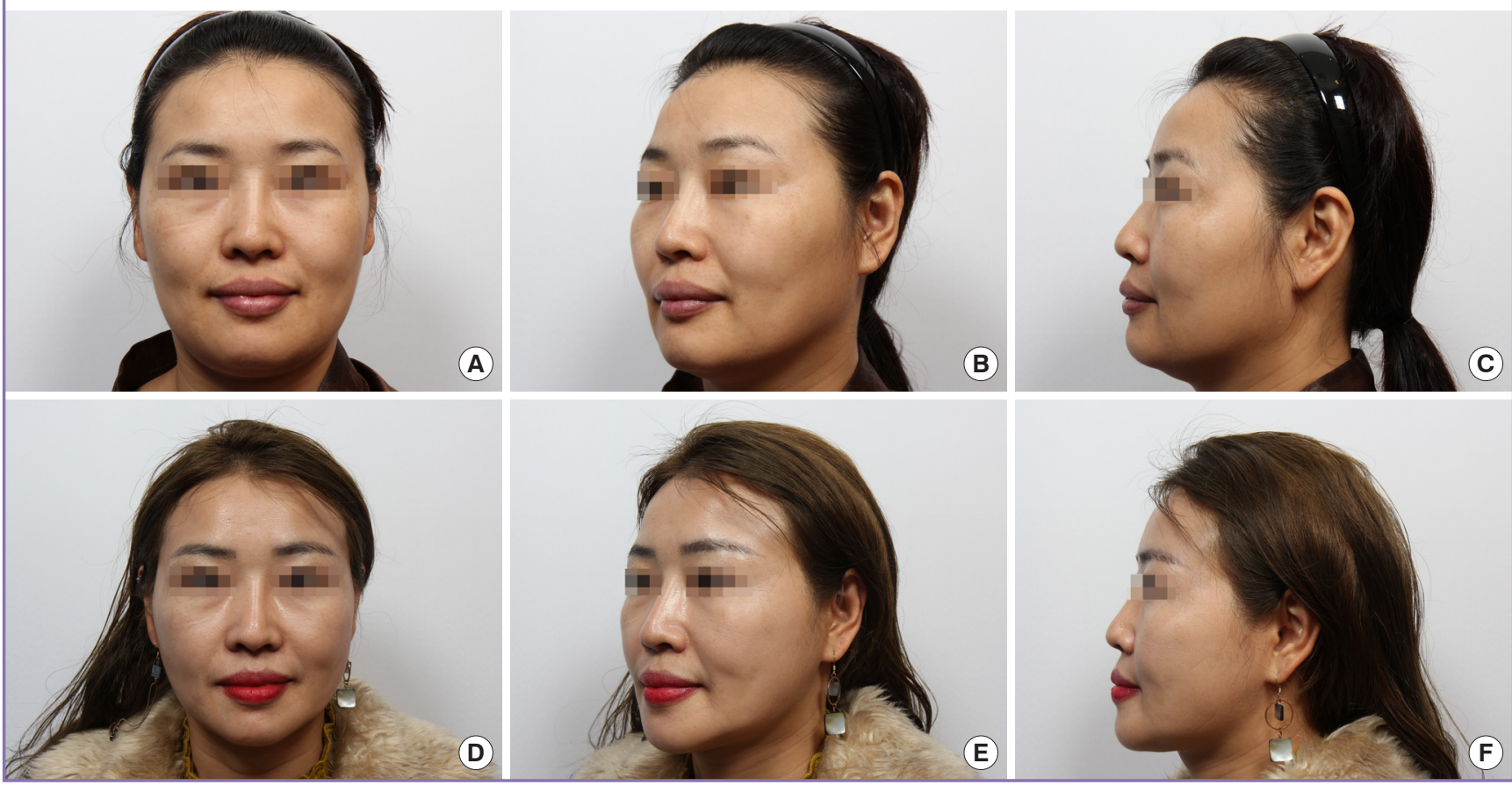

\section{Table 2. Postoperative complications}

\begin{tabular}{|lc|}
\hline Complications & No. (\%) \\
\hline Prolonged edema over 3 weeks & $7(7.4)$ \\
Skin dimpling or depression & $3(3.2)$ \\
Thread protrusion & $1(1.1)$ \\
Overcorrected lipodystrophy & $2(2.1)$ \\
Undercorrected lipodystrophy & $6(6.4)$ \\
Seroma or hematoma & 0 \\
Visible scar & 0 \\
\hline
\end{tabular}

recovery time and undercorrection, and the most severe complaints were caused by depression as a result of overcorrection. Two patients who underwent additional fat grafting due to overcorrection stated that they were very dissatisfied and accounted for two-thirds (67\%) of the very dissatisfied patients.

All postoperative complications resolved successfully. There were no additional late complications at the time of the satisfaction survey, which was conducted at least 6 months after surgery.

\section{DISCUSSION}

Within the framework of the horizontal thirds concept, the lower face refers to the area from the subnasale to the menton. The lower face can be divided into the facial aesthetic units, which
Table 3. Patients' satisfaction rate

\begin{tabular}{|lc|}
\hline Satisfaction & No. \\
\hline Very satisfied & 19 \\
Satisfied & 26 \\
Unsatisfied $^{\text {a) }}$ & 6 \\
Very unsatisfied & 3 \\
\hline a) Unsatisfied group: 3 cases due to prolonged recovery time, 3 cases due to \\
undercorrection. \\
b)Very unsatisfied group: 2 cases due to overcorrected lipodystrophy, 1 case due \\
to skin dimpling resulting from thread lift. \\
\hline
\end{tabular}

include the upper and lower lip units, the buccal and lateral cheek units, and the mental unit. To create a beautiful lower face, achieving symmetry, balance, and harmony between these units is as important as shaping each individual unit $[2,3]$.

Although conventional facelift procedures may provide excellent and long-lasting results [4], demand is growing for less invasive procedures because of the relatively high cost of facelift procedures, the long downtime, and fear of facelift-related complications [5]. Because patients expect excellent outcomes from procedures that avoid the disadvantages of a facelift, it is challenging to satisfy their needs through a single method; therefore, various techniques should be combined and individualized for each patient.

Common minimally invasive procedures to improve the aesthetics of the lower face include BoNTA injection, injectable 
filler injection, LAL, fat grafting, SAL, and thread lifting.

In the past, surgical debulking of the hypertrophied masseter muscle was mostly used to resolve square faces resulting from hypertrophied masseter muscles [6]; however, as BoNTA has become more popular, it has rapidly replaced surgical options $[7,8]$.

Injectable fillers are prepared from various biological and synthetic materials and are used to augment volume or correct wrinkles in the face. Filler injections into the lower face are mostly administered to restore lip volume and chin projection, as well as to camouflage the prejowl sulcus [8].

In response to the growing demand for minimally invasive procedures, laser-based procedures to decrease the amount of subcutaneous fat have been developed. LAL destroys the target tissue (i.e., fat cells) by inflicting thermal damage. Additionally, thermal stimulation by lasers can induce partial lifting effects and enhance facial skin appearance by causing skin contraction and collagen remodeling. Although 1,064-nm and 1,320-nm Qswitched Nd:YAG lasers are popular for lipolysis, they have been reported to have lower specificity for fat cells [9]. In contrast, 1,444-nm Nd:YAG lasers have a higher absorption rate for fat cells than lasers of other wavelengths [10-13].

SAL, a traditional method for lipolysis, has synergistic effects when combined with LAL [14]. As a result of the tunneling performed for the laser cannula and lipolysis, a liposuction cannula can be inserted easily and smoothly, which removes the fat cells disrupted by LAL along with any residual fat.

The position and volume of the facial soft tissue are altered by animation; therefore, a careful choice must be made regarding the fat to be removed and preserved based on appropriate consideration of the movement of soft tissues with and without facial expressions [15]. In our method, we first observed the areas where fat would be removed at various angles with the patient having a neutral facial expression, then observed changes in the facial tissue accompanying the movement of the facial muscles as the patient smiled, and finally decided on the area of fat removal. It is especially important to remove fat tissue conservatively in the middle cheek area, because a hollow could otherwise occur.

Thread lifting has emerged as a topic of widespread interest as a reliable and simple procedure for facial rejuvenation that avoids the shortcomings of traditional facelift techniques [16]. It became popular in the early 2000s following the introduction of Sulamanidze's antiptosis subdermal suspension suture (APTOS). However, the dramatic results reported in the study that introduced the APTOS lift were not reproducible, and questions about its longevity have consistently been raised [17]. Nevertheless, diverse types of threads have been developed, dif- fering in terms of material, thickness, the presence and direction of a barb, and the presence of cones.

After thread insertion, the tissues are lifted by the resistance created by the barb in the short term, but in the long term, the lift is sustained by inducing fibrotic adhesion. However, the degree of adhesion resulting from a thread lift is considered to be too weak to maintain the lifted tissue in its new position, and most studies have suggested that thread lifting alone is highly limited in terms of effects and longevity $[16,18]$.

The BFP plays an important role in aesthetics and function, such as preventing the indrawing of the cheek during sucking, facilitating a sliding movement of adjacent muscles, and protecting neurovascular bundles [19]. Further, it is also used for various purposes in reconstructive and cosmetic surgery [20]. With aging, the borders of the premasseteric space become loose and the BFP begins to jut out of the masticator space [21]. The descended BFP and soft tissue descending from the midface form a hammock-like jowl between the platysma auricular fascia and the mandibular ligament, which is relatively firm. When mandibular resorption progresses due to aging, the mandibular angle becomes more obtuse, which makes the jowl and prejowl sulcus more prominent [22]. A protruding, descended BFP is treated by making a $1-\mathrm{cm}$ intraoral incision, through which the buccinator is split to approach the BFP and remove the fat with mild traction. It is imperative for the BFP to be conservatively removed, as excessive removal causes a hollow middle cheek.

Since an aesthetically unpleasing appearance of the lower face is multifactorial, aesthetic correction of the lower face using a single method is difficult. Therefore, plastic surgeons often utilize various methods to treat patients [14,21,23-25].

There are some benefits of performing lipolysis followed by a thread lift. The preceding lipolysis procedure increases the mobility of the skin flap from the underlying tissue, making skin elevation easier than when performing a thread lift alone. Furthermore, by elevating an undermined skin flap through multiple cannulation, wide adhesion between the skin flap and underlying tissue can be induced, thereby prolonging the longevity of the thread lift. By reducing the amount of fat in the lower face prior to thread lifting, the weight to be supported by the thread is reduced, which prolongs the effects of lifting. Therefore, in our technique, thread lifting may result in the skin flap being better maintained through wound adhesion during the early wound healing process, in addition to face lifting. To maintain the lifting effect in the early postoperative period, the thread should have cogs to grasp the lifted tissue and a length over 15 $\mathrm{cm}$ to reach the lower facial tissue from the temporal hairline. The scar tissue between the skin flap and underlying tissue can play the role of a facial ligament; thus, the lifting effects will per- 
sist even after the thread is absorbed.

In our study, prolonged edema subsided spontaneously without any intervention within 1 month postoperatively. To reduce postoperative edema, it is essential to minimize bleeding. We injected a sufficient amount of the tumescent solution and used a thin cannula ( $1.8 \mathrm{~mm}$ or $2.0 \mathrm{~mm}$ ) for facial liposuction. Skin dimpling at the distal end of the PDO thread occurred in two patients. In one patient, the dimpling was relieved with a light massage, and in the other it was alleviated by a manual massage and a subcision using an 18-gauge needle. If the proximal end of a thread protruded, it was pulled out and cut. Skin dimpling or depression may be caused by a superficial injection of the thread. During thread insertion, the end of the thread should be located in a deeper layer of the subcutaneous plane. If a patient complained of undercorrection, additional LAL and SAL were performed, and if the middle cheek compartment was overcorrected, fat grafting was carried out on the depressed cheek. Our multi-modal treatment strategy is suitable for younger patients ( $<50$ years of age), those with a thin and pliable skin texture, and those with localized fatty accumulations in the lower face and submandibular area.

Better outcomes can be achieved by understanding the advantages and disadvantages of various methods of attaining aesthetic enhancement of the lower face and appropriately combining various techniques. However, to further demonstrate the effectiveness of our method, a quantitative analysis will be beneficial in future studies.

\section{NOTES}

\section{Conflict of interest}

No potential conflict of interest relevant to this article was reported.

\section{Ethical approval}

The study was approved by the Institutional Review Board of Eulji University Eulji Medical Center (IRB No. EMCIRB 201910-004) and performed in accordance with the principles of the Declaration of Helsinki. Written informed consents were obtained.

\section{Patient consent}

The patients provided written informed consent for the publication and the use of their images.

\section{Author contribution}

Conceptualization: TK Jeong, KH Min. Data curation: TK Jeong. Formal analysis: KH Min. Methodology: TKJeong, CH
Chung, KH Min. Project administration: KH Min. Writing original draft: $\mathrm{CH}$ Chung, $\mathrm{KH}$ Min. Writing - review \& editing: CH Chung.

\section{ORCID}

Tae Kwang Jeong https://orcid.org/0000-0003-0285-2410

Chang Ho Chung https://orcid.org/0000-0002-9431-737X

Kyung Hee Min https://orcid.org/0000-0002-3456-0191

\section{REFERENCES}

1. Floyd EM, Sukato DC, Perkins SW. Advances in face-lift techniques, 2013-2018: a systematic review. JAMA Facial Plast Surg 2019;21:252-9.

2. Swift A, Remington K. BeautiPHIcation(TM): a global approach to facial beauty. Clin Plast Surg 2011;38:347-77.

3. Fattahi TT. An overview of facial aesthetic units. J Oral Maxillofac Surg 2003;61:1207-11.

4. Hashem AM, Couto RA, Duraes EFR, et al. Facelift part I: history, anatomy, and clinical assessment. Aesthet Surg J 2020;40:1-18.

5. Cosmetic Surgery National Data Bank Statistics. Aesthet Surg J 2017;37(suppl_2):1-29.

6. Bocchialini G, Castellani A, Negrini S, et al. New management in bilateral masseter muscle hypertrophy. Craniomaxillofac Trauma Reconstr 2017;10:325-8.

7. Liew S, Dart A. Nonsurgical reshaping of the lower face. Aesthet Surg J 2008;28:251-7.

8. de Maio M, Wu WTL, Goodman GJ, et al. Facial assessment and injection guide for botulinum toxin and injectable hyaluronic acid fillers: focus on the lower face. Plast Reconstr Surg 2017;140:393e-404e.

9. Khoury JG, Saluja R, Keel D, et al. Histologic evaluation of interstitial lipolysis comparing a 1064, 1320 and $2100 \mathrm{~nm}$ laser in an ex vivo model. Lasers Surg Med 2008;40:402-6.

10. Tark KC, Jung JE, Song SY. Superior lipolytic effect of the 1,444 nm Nd:YAG laser: comparison with the 1,064 nm Nd:YAG laser. Lasers Surg Med 2009;41:721-7.

11. Jung SK, Jang HW, Kim HJ, et al. A prospective, long-term follow-up study of 1,444 nm Nd:YAG laser: a new modality for treating axillary bromhidrosis. Ann Dermatol 2014;26: 184-8.

12. Tagliolatto S, Medeiros VB, Leite OG. Laserlipolysis: update and literature review. Surg Cosmet Dermatol 2012;4:16474.

13. Lim SD, Youn JI, Kim WS, et al. Comprehensive histologic analysis of interstitial lipolysis with the $1444 \mathrm{~nm}$ wavelength during a 3-month follow-up. Histol Histopathol 2011;26: 
1375-82.

14. Sun Y, Wu SF, Yan S, et al. Laser lipolysis used to treat localized adiposis: a preliminary report on experience with Asian patients. Aesthetic Plast Surg 2009;33:701-5.

15. Rawlani R, Qureshi H, Rawlani V, et al. Volumetric changes of the mid and lower face with animation and the standardization of three-dimensional facial imaging. Plast Reconstr Surg 2019;143:76-85.

16. Gulbitti HA, Colebunders B, Pirayesh A, et al. Thread-lift sutures: still in the lift? A systematic review of the literature. Plast Reconstr Surg 2018;141:341e-347e.

17. Villa MT, White LE, Alam M, et al. Barbed sutures: a review of the literature. Plast Reconstr Surg 2008;121:102e-108e.

18. Fukaya M. Two mechanisms of rejuvenation using thread lifting. Plast Reconstr Surg Glob Open 2018;6:e2068.

19. Yousuf S, Tubbs RS, Wartmann CT, et al. A review of the gross anatomy, functions, pathology, and clinical uses of the buccal fat pad. Surg Radiol Anat 2010;32:427-36.
20. Kim JT, Sasidaran R. Buccal fat pad: an effective option for facial reconstruction and aesthetic augmentation. Aesthetic Plast Surg 2017;41:1362-74.

21. Frisenda JL, Nassif PS. Correction of the lower face and neck. Facial Plast Surg 2018;34:480-7.

22. Wong $\mathrm{CH}$, Mendelson B. Newer understanding of specific anatomic targets in the aging face as applied to injectables: aging changes in the craniofacial skeleton and facial ligaments. Plast Reconstr Surg. 2015 Nov;136(5 Suppl):44S-48S.

23. Doerr TD. Lipoplasty of the face and neck. Curr Opin Otolaryngol Head Neck Surg 2007; 15:228-32.

24. Wilson AJ, Taglienti AJ, Chang CS, et al. Current applications of facial volumization with fillers. Plast Reconstr Surg 2016;137:872e-889e.

25. Chang CS, Kang GC. Achieving ideal lower face aesthetic contours: combination of tridimensional fat grafting to the chin with masseter botulinum toxin injection. Aesthet Surg J 2016;36:1093-100. 